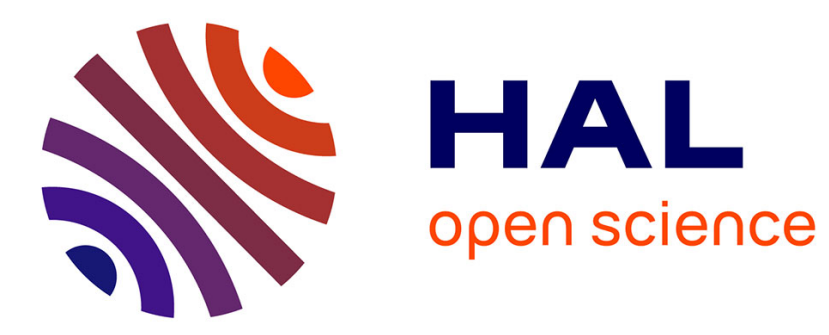

\title{
A High Frequency Equivalent Circuit and Parameter Extraction Procedure for Common Mode Choke in the EMI Filter
}

\author{
Wenhua Tan, Carlos Cuellar, Xavier Margueron, Nadir Idir
}

\section{- To cite this version:}

Wenhua Tan, Carlos Cuellar, Xavier Margueron, Nadir Idir. A High Frequency Equivalent Circuit and Parameter Extraction Procedure for Common Mode Choke in the EMI Filter. IEEE Transactions on Power Electronics, 2013, 28 (3), pp.1157 - 1166. 10.1109/TPEL.2012.2209206 hal-01886770

\author{
HAL Id: hal-01886770 \\ https://hal.science/hal-01886770
}

Submitted on 3 Oct 2018

HAL is a multi-disciplinary open access archive for the deposit and dissemination of scientific research documents, whether they are published or not. The documents may come from teaching and research institutions in France or abroad, or from public or private research centers.
L'archive ouverte pluridisciplinaire HAL, est destinée au dépôt et à la diffusion de documents scientifiques de niveau recherche, publiés ou non, émanant des établissements d'enseignement et de recherche français ou étrangers, des laboratoires publics ou privés. 


\title{
A High Frequency Equivalent Circuit and Parameter Extraction Procedure for Common Mode Choke in EMI Filter
}

\author{
Wenhua Tan, Carlos Cuellar, Member, IEEE, Xavier Margueron, Member, IEEE, and Nadir Idir, Member, IEEE
}

\begin{abstract}
Power converters with high switching frequency generate conducted electromagnetic interference (EMI) noise. EMI filters are thus widely used to reduce these conducted noises for the compliance with electromagnetic compatibility standards. In this paper, a high frequency (HF) equivalent circuit model for common mode (CM) chokes used in EMI filters is proposed together with its parameter extraction procedure. This procedure is based on impedance measurements and it incorporates an iterative rational function approximation fitting algorithm to extract the parameters in the model. The proposed model and procedure is applied to a planar $\mathrm{CM}$ choke which is used to realize an EMI filter. The simulated results of the filter show good agreement with the experimental ones. This extraction procedure is quite general and it can also be extended to identify the HF model of other passive components.
\end{abstract}

Index Terms-Common mode choke, electromagnetic interference filter, equivalent circuit, rational function approximation.

\section{INTRODUCTION}

$\mathrm{E}$ LECTROMAGNETIC interference (EMI) filters are commonly used solutions for mitigating the conducted emissions produced by power converters [1]. A typical topology of EMI filter with ideal components is shown in Fig. 1. However, a real EMI filter is much more complicated and its performances depend on many factors such as filter topology, magnetic material, grounding method, stray elements of components, and parasitic coupling between components etc. [2]-[4]. Complete physic-based models of an EMI filter or their components require extensive electromagnetic analysis, so they are very complex and

Manuscript received February 6, 2012; revised March 30, 2012 and May 25, 2012; accepted July 2, 2012.

W. Tan and X. Margueron are with the Laboratory of Electrical Engineering and Power Electronics of Lille, Ecole Centrale de Lille, Villeneuve d'Ascq, France (e-mail: wenhua.tan@ec-lille.fr; xavier.margueron@ec-lille.fr).

C. Cuellar and N. Idir are with the Laboratory of Electrical Engineering and Power Electronics of Lille, Université de Lille 1, Villeneuve d'Ascq, France, (e-mail: carlos.cuellar@univ-lille1.fr; nadir.idir@univ-lille1.fr).

Color versions of one or more of the figures in this paper are available online at $\mathrm{http}: / /$ ieeexplore.ieee.org.

Digital Object Identifier

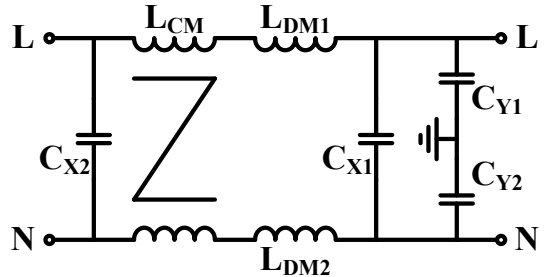

Fig. 1. Typical topology of EMI filter

specific to limited applications [5], [6]. Modeling methods based on measurements are more general, direct and accurate for describing the characteristics of the device under study.

The insertion loss (IL) of an EMI filter is usually measured with $50 \Omega / 50 \Omega$ convention. However, the IL of the filter under working condition depends on the impedances of the source and the load, which vary with frequency [7], [8]. In order to obtain the IL of the filter, many solutions have been reported. In [9], a four-port measurement method using vector network analyzer is presented. This method consists in a black-box modeling of the whole filter with mixed-mode S-parameters. By post-processing the obtained S-parameter data, the IL of the filter can hence be calculated with any source and load impedances. In [10], a modal model of common mode (CM) chokes based on four-port S-parameter measurements is presented. Though modal models enable to analyze the conversion of the noise between differential mode (DM) and $\mathrm{CM}$, they also require compatible modal model of noise sources for simulations, which complicates the modeling process. An alternative is to use equivalent circuit models, which are physic based and are compatible with most of the simulation tools. In [11], the equivalent circuit of a DM EMI filter is identified by S-parameters measurements. This approach can correctly extract the parasitic couplings in the filter, resulting in good modeling precision. Impedance measurement is a more frequently used technique which has long been studied for identifying the equivalent circuits of passive magnetic components [12], [13]. Recently, a lumpedelement high frequency (HF) model for CM chokes has been proposed [14], [15]. This model can be easily built by extracting the parameters from impedance measurement results and can effectively describe the HF characteristics of the studied CM choke and EMI filter. However, the topology 


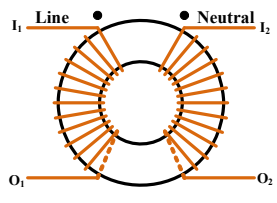

(a)

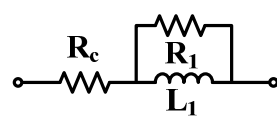

(c)

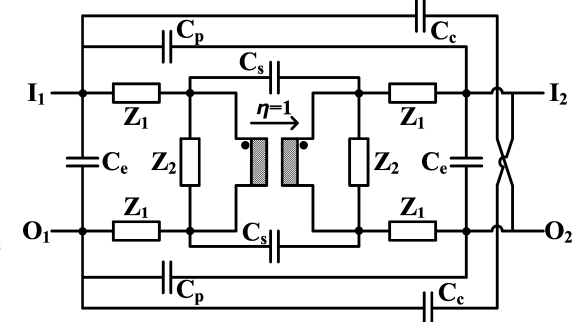

(b)

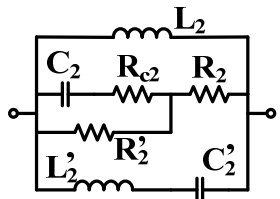

(d)

Fig. 2. HF circuit model for CM chokes proposed in [15]. (a) Toroidal CM choke. (b) HF equivalent circuit. (c) Equivalent circuit of leakage impedance $Z_{1}$. (d) Equivalent circuit of magnetizing impedance $Z_{2}$.

of the HF model is chosen heuristically, which requires lots of experience and tests. Moreover, the extraction procedure derives the parameters of the model by observing the impedance curves, leading to quite time-consuming trial/error iterations.

To simplify the extraction process, numerical methods can be applied. In [16], a genetic algorithm is used to extract the behavior model of chokes, yielding an accurate and reliable broadband equivalent circuit. Nevertheless, the convergence time of such algorithm is usually long when the problem has a large search space. In [17], a physics-based equivalent circuit of $\mathrm{CM}$ chokes is built with a rational function approximation (RFA) method based on linear optimization [18]. However, the topology of the equivalent circuit is predefined so prior knowledge about the component is needed.

In this study, a HF model of CM chokes is proposed together with a systematic extraction procedure. This procedure is aided by the RFA method incorporating an iterative scheme (referred as IRFA) and it allows a fast and accurate extraction of the parameters in the model. This paper is organized as follows. In Section II, the HF model introduced in [15] is reviewed. Next, the RFA and the proposed IRFA methods are presented in Section III. In Section IV, the proposed HF model of CM chokes and its extraction procedure are introduced and then applied to a planar CM choke. Finally, experimental verifications of the procedure as well as some discussion of the results are given in Section V. Section VI concludes this paper.

\section{HF MODEL OF CM CHOKES}

\section{A. Review of the Existing HF Model for CM Chokes}

The equivalent circuit for toroidal $\mathrm{CM}$ chokes proposed in [15] [see Fig. 2(a)] is illustrated in Fig. 2(b). As seen, it is a symmetrical structure and the transformation ratio $\eta$ is assumed to be unity. This model can describe the behavior of many types of CM chokes as long as the winding symmetry is satisfied. The leakage impedance $Z_{1}$ includes the winding

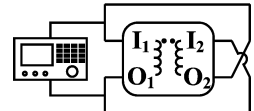

(a)

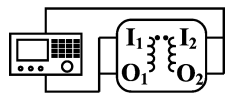

(d)

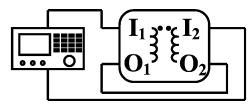

(b)

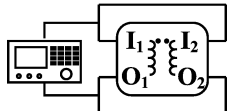

(c)

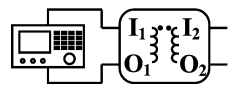

(e)
Fig. 3. Impedance measurement configurations for the extraction. (a) $\mathrm{T}_{0}$. (b) $T_{1}$. (c) $T_{2}$. (d) $T_{3}$. (e) $T_{4}$.

resistance, the leakage inductance and their variations due to skin and proximity effects of the winding conductor [see Fig. 2(c)]. The magnetizing impedance $Z_{2}$ describes the behavior of magnetic core, including the frequency-dependent inductance and losses [see Fig. 2(d)]. In addition, $C_{\mathrm{e}}$ stands for the self-parasitic capacitance of each winding whereas the sum $C_{\mathrm{s}}+C_{\mathrm{p}}+C_{\mathrm{c}}$ is related to the inter-winding capacitances of the component. Examining the admittance $Y_{2}=\left(Z_{2}\right)^{-1}$, it is found that $Y_{2}$ contains:

1) One pole at origin: the branch of $L_{2}$;

2) One stable real pole: the branch of $R_{2}, R_{\mathrm{C} 2}, C_{2}$ and $R_{2}^{\prime}$;

3) One pure imaginary pole-pair: the branch of $L_{2}^{\prime}$ and $C_{2}^{\prime}$.

With these elements, the HF behavior of the magnetizing impedance $Z_{2}$ is accurately modeled.

\section{B. Method of Parameter Extraction}

To identify the parameters of the model shown in Fig. 2(b), five selected impedance measurements are carried out, as presented in Fig. 3. All the parameters of the model are obtained through these impedance measurements, as summarized below:

1) $\mathrm{T}_{0}, \mathrm{~T}_{1}, \mathrm{~T}_{2}$ and $\mathrm{T}_{3}$ : parasitic capacitances $C_{\mathrm{c}}, C_{\mathrm{e}}, C_{\mathrm{p}}$ and $C_{\mathrm{s}}$;

2) $T_{0}$ and $T_{1}$ : leakage impedance $Z_{1}$;

3) $T_{2}$ and $T_{4}$ : magnetizing impedance $Z_{2}$.

The extraction procedure is based on observations of the impedance curves with some typical frequency responses, e.g., $20 \mathrm{~dB} /$ Dec as inductance and $-20 \mathrm{~dB} /$ Dec as capacitance. The values of the parasitic capacitances are calculated using the resonance frequencies. To fit the model with the measurement results, some manual adjustments of the parameters are needed for $Z_{1}$ and $Z_{2}$.

Though good accuracy is achieved in [15], the HF model shown in Fig. 2(b) can be improved for the following reasons: 1) The extraction procedure requires manual adjustments on the parameters of the model to achieve a desired accuracy. As a result, the procedure is complex and time-consuming. Therefore, a computer-aided procedure is preferable.

2) The topology of the equivalent circuit is determined according to experimental observations. For example, the admittance $Y_{2}=1 / Z_{2}$ is chosen to have one stable pole and one imaginary pole-pair from experience. In consequence, a systematic equivalent circuit synthesis method is needed to choose the topology.

In view of these necessities, a HF equivalent circuit model for $\mathrm{CM}$ chokes and an IRFA based extraction procedure are 
proposed to facilitate the process. The IRFA method is first presented in the next section.

\section{PARAMETER EXTRACTION USING ItERATIVE RATIONAL FUNCTION APPROXIMATION}

\section{A. Brief Review of Rational Function Approximation Method}

The impedance $Z(s)$ (or admittance $Y(s)$ ) of a linear circuit network can be represented by a rational function.

$$
Z(s)=\frac{N(s)}{D(s)}=\frac{b_{m} s^{m}+b_{m-1} s^{m-1}+\cdots+b_{1} s+b_{0}}{a_{n} s^{n}+a_{n-1} s^{n-1}+\cdots+a_{1} s+1}
$$

with $|m-n| \leq 1$ and $s=j \omega$. Without loss of generality, only the impedance $Z(s)$ is considered in this section. The same analysis is valid for the admittance $Y(s)$. The goal of the RFA is to search the values of $a_{i}$ and $b_{i}$ to minimize the error between the rational function $Z(s)$ and measured data $Z_{\text {meas }}(s)$, as expressed by:

$$
\arg \min _{a_{i}, b_{i}} \sum_{k}\left|Z_{\text {meas }}\left(s_{k}\right)-Z\left(s_{k}\right)\right|^{2} \Rightarrow Z_{\text {meas }}\left(s_{k}\right)=\frac{N\left(s_{k}\right)}{D\left(s_{k}\right)} .
$$

where $s_{k}=j \omega_{k}$. To handle this nonlinear optimization problem, Levy used a linearization technique [19]. Stemming from Levy's method, the authors introduced in [18] the RFA method for generating the macro-model of HF interconnects. Reformulating (2) by multiplying $D\left(s_{k}\right)$ gives:

$$
N\left(s_{k}\right)-Z_{\text {meas }}\left(s_{k}\right) D\left(s_{k}\right)=0 .
$$

Separating the real parts and imaginary parts of (3) yields a linear system of $a_{i}$ and $b_{i}$ :

$$
\left\{\begin{array}{l}
\operatorname{Re}\left[N\left(s_{k}\right)\right]-\operatorname{Re}\left[Z_{\text {meas }}\left(s_{k}\right) D\left(s_{k}\right)\right]=0 \\
\operatorname{Im}\left[N\left(s_{k}\right)\right]-\operatorname{Im}\left[Z_{\text {meas }}\left(s_{k}\right) D\left(s_{k}\right)\right]=0
\end{array} .\right.
$$

This system is over-determined due to the large number of measured points. In order to calculate the unknowns $a_{i}$ and $b_{i}$, a least-square method using $\mathrm{QR}$ factorization is applied to solve the over-determined system (4). Once the values of $b_{i}$ are known, the poles of (1) can be derived by solving the roots of the denominator $D(s)$. The impedance $Z(s)$ can then be written into a pole-residue form:

$$
Z(s)=d+e \cdot s+\sum_{i=1}^{n} \frac{r_{i}}{s-p_{i}} .
$$

It should be noted that $r_{i}$ and $p_{i}$ can be complex-valued. Again, the terms $d$, $e$, and $r_{i}$ are solved from this linear overdetermined system (5) by the least-square method [18].

\section{B. Iterative Rational Function Approximation Method}

The RFA method is proven to be efficient with noise-free simulated data. However it cannot be directly employed for our application, since Levy's linearization technique is known to have a frequency bias problem [20]. In (2), $Z_{\text {meas }}\left(s_{k}\right)$ is multiplied by $D\left(s_{k}\right)$ that increases rapidly with $s_{k}$ (as frequency increases). This means the errors of (2) are biased by $D\left(s_{k}\right)$, making HF errors more important than low frequency (LF) errors. As a result, this frequency bias due to $D\left(s_{k}\right)$ causes poor fitting precision at LF. However, to correctly identify elements like DC winding resistance of CM chokes, this lack

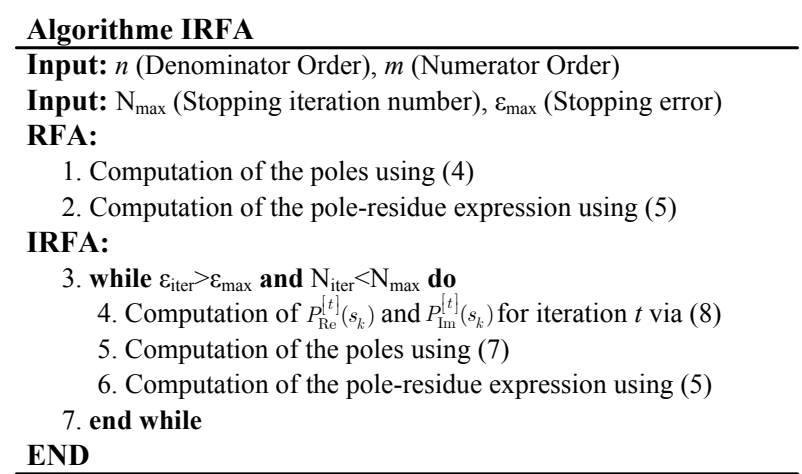

Fig. 4. IRFA algorithm.

of fitting precision at LF is undesirable. To handle the frequency bias, the RFA method is improved in this work using the Sanathanan-Koerner (SK) iteration [19]. The denominator of the iteration $t-1 D^{[t-1]}\left(s_{k}\right)$ is divided on both sides of (3) for the iteration $t$, giving

$$
\frac{N^{[t]}\left(s_{k}\right)}{D^{[t-1]}\left(s_{k}\right)}-\frac{Z_{\text {meas }}\left(s_{k}\right) D^{[t]}\left(s_{k}\right)}{D^{[t-1]}\left(s_{k}\right)}=0 .
$$

It can be seen that the bias due to $D^{[t]}\left(s_{k}\right)$ is alleviated by dividing $D^{[t-1]}\left(s_{k}\right)$, resulting in better fitting accuracy at LF. Based on (4) and (6), the proposed IRFA formulation used in this work is given by

$$
\left\{\begin{array}{l}
\operatorname{Re}\left[P_{\mathrm{Re}}^{[t]}\left(s_{k}\right) N^{[t]}\left(s_{k}\right)\right]-\operatorname{Re}\left[P_{\mathrm{Re}}^{[t]}\left(s_{k}\right) Z_{\text {meas }}\left(s_{k}\right) D^{[t]}\left(s_{k}\right)\right]=0 \\
\operatorname{Im}\left[P_{\operatorname{Im}}^{[t]}\left(s_{k}\right) N^{[t]}\left(s_{k}\right)\right]-\operatorname{Im}\left[P_{\operatorname{Im}}^{[t]}\left(s_{k}\right) Z_{\text {meas }}\left(s_{k}\right) D^{[t]}\left(s_{k}\right)\right]=0
\end{array} .\right.
$$

with the weighting factors $P_{\mathrm{Re}}^{[t]}\left(s_{k}\right)$ and $P_{\mathrm{Im}}^{[t]}\left(s_{k}\right)$ given by

$$
\left\{\begin{array}{l}
P_{\mathrm{Re}}^{[t]}\left(s_{k}\right)=\frac{\left[D^{[t-1]}\left(s_{k}\right)\right]^{*}}{\operatorname{Re}\left[D^{[t-1]}\left(s_{k}\right)\right] \operatorname{Re}\left[N^{[t-1]}\left(s_{k}\right)\right]+\operatorname{Im}\left[D^{[t-1]}\left(s_{k}\right)\right] \operatorname{Im}\left[N^{[t-1]}\left(s_{k}\right)\right]} \\
P_{\operatorname{Im}}^{[t]}\left(s_{k}\right)=\frac{\left[D^{[t-1]}\left(s_{k}\right)\right]^{*}}{\operatorname{Re}\left[D^{[t-1]}\left(s_{k}\right)\right] \operatorname{Im}\left[N^{[t-1]}\left(s_{k}\right)\right]-\operatorname{Im}\left[D^{[t-1]}\left(s_{k}\right)\right] \operatorname{Re}\left[N^{[t-1]}\left(s_{k}\right)\right]}
\end{array}\right.
$$

It should be mentioned that in $P_{\mathrm{Re}}^{[t]}\left(s_{k}\right)$ and $P_{\mathrm{Im}}^{[t]}\left(s_{k}\right)$, a balanced weighting is used to achieve comparable fitting precision for both real parts and imaginary parts. The derivation of (8) is detailed in Appendix. The algorithm of IRFA is given in Fig. 4, where the $\varepsilon_{\text {iter }}$ is defined as the maximum error

$$
\varepsilon_{\text {iter }}=\max _{k}\left\{\left|Z_{\text {fit }}\left(j \omega_{k}\right)-Z_{\text {meas }}\left(j \omega_{k}\right)\right| /\left|Z_{\text {meas }}\left(j \omega_{k}\right)\right|\right\} .
$$

The RFA method is first performed to provide a starting point, the IRFA is then applied until the stopping condition(s) (in this work: $\varepsilon_{\max }=10 \%, \mathrm{~N}_{\max }=15$ ) are fulfilled. To show the efficiency of the proposed IRFA algorithm, different methods are applied to fit the measured impedance (from $40 \mathrm{~Hz}$ to 70 $\mathrm{MHz}$ ) of an 8-turn planar inductor with Ferroxcube 3F3Planar E38 ferrite core. A rational function like (1) with $m=n$ $=6$ is used for the fitting. It can be seen on Fig. 5(a) that the RFA fitting results are wrong on LF due to the frequency bias problem and the errors decrease when frequency increases. In 

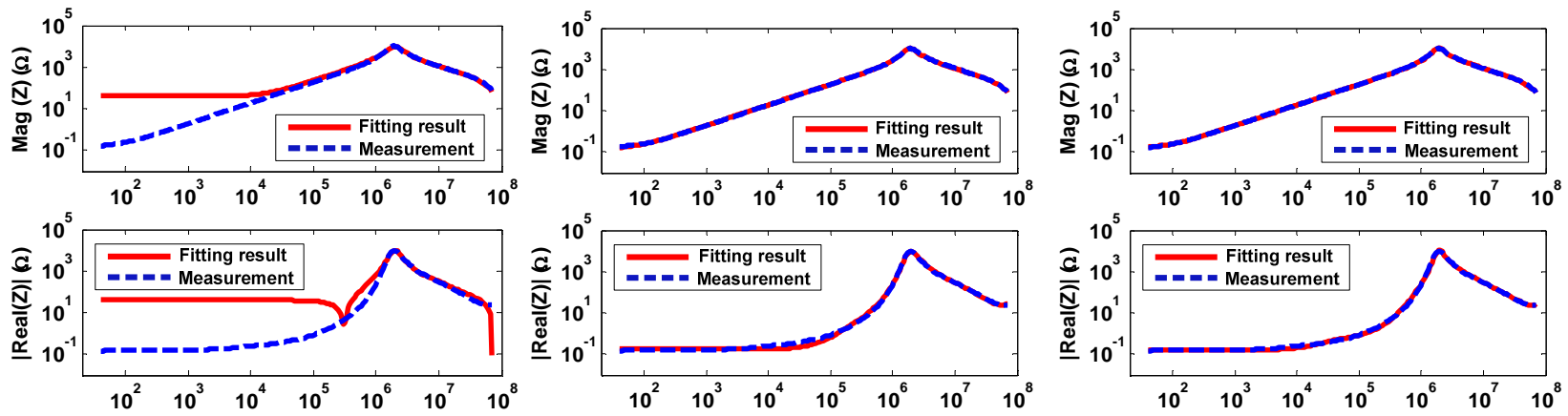

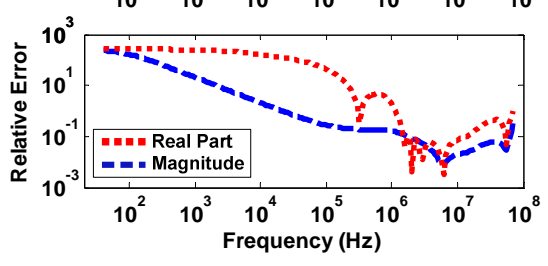

(a)

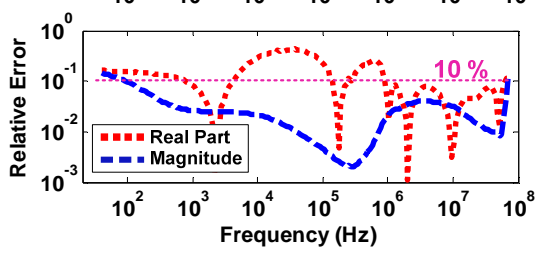

(b)

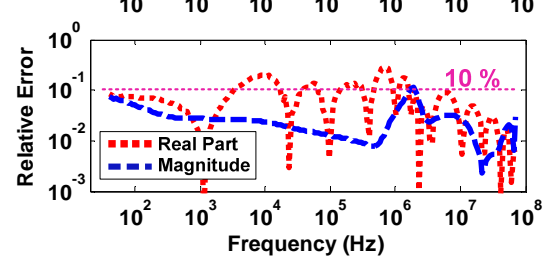

(c)

Fig. 5. Comparison of the fitting results. (a) RFA with frequency bias problem. (b) IRFA without balanced weighting. (c) IRFA with balanced weighting.

Fig. 5(b) and (c), the IRFA methods alleviate the frequency bias problem in Fig. 5(a). As seen, better fitting accuracies are achieved at LF. The difference between Fig. 5(c) and (b) is the implementation of balanced weighting. It can be seen from the error curve shown in Fig. 5(c) that with balanced weighting, the fitting accuracy of the real part is further improved in comparison with Fig. 5(b). The precision of IRFA fitting is very good on wide frequency band because the impedance curve is quite smooth (very few resonances). However, the error may exceed the specified error level $\varepsilon_{\max }$ on the two extremities of fitting range and the resonance frequencies. To achieve a better precision, one can increase the order of the rational function at the expense of obtaining a more bulky equivalent circuit. In order to obtain a simple equivalent circuit, the choice of the order turns out to be a trade-off between the accuracy and the complexity of the circuit.

\section{Equivalent Circuit Synthesis}

Based on the results obtained by the IRFA method, a systematic approach is used for synthesizing the equivalent circuit [21]. Representing (5) by the Foster expansion:

$$
Z(s)=d+e \cdot s+\underbrace{\sum_{i=1}^{N_{r}} \frac{r_{i}}{s-p_{i}}}_{\text {real poles }}+\underbrace{\sum_{i=1}^{N_{c}}\left(\frac{a_{i} s+b_{i}}{s^{2}+m_{i} s+n_{i}}\right)}_{\text {complex conjugate pole pairs }} .
$$

Note that the $a_{i}, b_{i}, m_{i}$, and $n_{i}$ are different variables from those used in (1). As seen, the $Z(s)$ can be expressed by the sum of a constant term $d$, an $s$-proportional term $e$, real poleresidue terms and complex conjugate pole-residue pair terms. The same expression holds for the admittance $Y(s)$. The constant $d$ corresponds to a resistor and the $e \cdot s$ term corresponds to a capacitor for $Y(s)$ or an inductor for $Z(s)$, respectively. In Table $I$, the detailed equivalent circuit synthesis methods for real-pole terms and complex-pole pair terms are summarized.
1) Real pole: With a positive residue $r_{i}$, the equivalent circuit is simply a $R-L$ in series for $Y(s)$ or $R-C$ in parallel for $Z(s)$, as shown in Table I. However, negative $r_{i}$ may appear in the IRFA results, resulting in negative valued elements. Strictly speaking, negative elements are not feasible in real circuit design so that they should be avoided. However, for EMI modeling and simulation purposes, these elements can be accepted for synthesizing an accurate equivalent circuit. Nonetheless, the negative elements are difficult to handle during time-domain simulations. To circumvent this difficulty, we suggest another topology: a positive-valued cell together with a negative resistance, as illustrated in Table I. Suppose that a negative residue $r_{i}$ is extracted with a stable real pole $p_{i}$ $\left(p_{i}<0\right)$ for the admittance $Y(s)$. Normally, a negative R-L series cell is obtained. However, according to Table I, the circuit composed of a positive $R-C$ series cell in parallel with a negative resistance $-R$ can be used, where the expression for $R, C$ and $-R$ are given by

$$
\begin{cases}R=p_{i} / r_{i} & \text { positive } \\ C=-1 /\left(p_{i} R\right) & \text { positive } \\ -R=-p_{i} / r_{i} & \text { negative }\end{cases}
$$

This circuit is equivalent to the negative $R-L$ series cell since they share the same rational function. The negative resistance $-R$ are subsequently merged into the constant term $d$ in (10), resulting in a new constant term $d^{*}=d-1 / R=d-r_{i} / p_{i}$. Similar analysis can be done for the case of impedance $Z(s)$. With these circuits, negative elements for real pole terms can be reduced to minimum.

2) Complex-pole pair: To treat complex-pole pair, a fourelement circuit can be used [21]. It is the minimal type realization since there are four free variables $a_{i}, b_{i}, m_{i}$ and $n_{i}$ in the rational function (see Table I). Another equivalent circuit referred as "Extended" type, uses a six-element circuit but with easier parameter calculation formulas [22]. In Fig. 6, the 
TABLE I

EQUiVAlENT CiRCUIT SyNTHESIS FOR REAL POLES AND COMPLEX-POLE PAIRS

\begin{tabular}{|c|c|c|c|c|c|}
\hline \multirow{2}{*}{\multicolumn{2}{|c|}{ Term }} & \multicolumn{2}{|c|}{$Y(s)$} & \multicolumn{2}{|c|}{$Z(s)$} \\
\hline & & Circuit & Expression & Circuit & Expression \\
\hline $\begin{array}{c}\text { Real pole } \\
r .\end{array}$ & $r_{i}>0$ & & $\begin{array}{l}L=1 / r_{i} \\
R=-p_{i} / r_{i}\end{array}$ & & $\begin{array}{l}C=1 / r_{i} \\
R=-r_{i} / p_{i}\end{array}$ \\
\hline$\frac{T_{i}}{s-p_{i}}$ & $r_{i}<0$ & $\|$ & $\begin{array}{l}R=p_{i} / r_{i} \\
C=-1 /\left(p_{i} R\right)\end{array}$ & & $\begin{array}{l}R=r_{i} / p_{i} \\
L=-R / p_{i}\end{array}$ \\
\hline $\begin{array}{c}\text { Complex-pole } \\
\text { Pair }\end{array}$ & $\begin{array}{l}\text { Minimal } \\
\text { Type }\end{array}$ & $\mathbf{R}_{1}$ & $\begin{array}{l}L=1 / a_{i} \\
R_{1}=L m_{i}-L^{2} b_{i} \\
R_{2}=n_{i} / b_{i}-R_{1} \\
C=1 /\left(b_{i} L R_{2}\right)\end{array}$ & R2 & $\begin{array}{l}C=1 / a_{i} \\
R_{2}=\left(C m_{i}-C^{2} b_{i}\right)^{-1} \\
R_{1}=\left(n_{i} / b_{i}-1 / R_{2}\right)^{-1} \\
L=R_{1} /\left(b_{i} C\right)\end{array}$ \\
\hline$\frac{a_{i} s+b_{i}}{s^{2}+m_{i} s+n_{i}}$ & $\begin{array}{c}\text { Extended } \\
\text { Type }\end{array}$ & $\mathrm{C}_{2}$ & $\begin{array}{l}R_{1}=n_{i} / b_{i}, \quad C_{1}=\frac{1}{m_{i} R_{1}} \\
L_{1}=\frac{1}{n_{i} C_{1}}, L_{2}=\frac{1}{a_{i}-1 / L_{1}} \\
R_{2}=m_{i} L_{2}, C_{2}=\frac{1}{n_{i} L_{2}}\end{array}$ & $H_{1}$ & $\begin{array}{l}R_{1}=b_{i} / n_{i}, L_{1}=R_{1} / m_{i} \\
C_{1}=\frac{1}{n_{i} L_{1}}, C_{2}=\frac{1}{a_{i}-1 / C_{1}} \\
R_{2}=\frac{1}{m_{i} C_{2}}, L_{2}=\frac{1}{n_{i} C_{2}}\end{array}$ \\
\hline
\end{tabular}

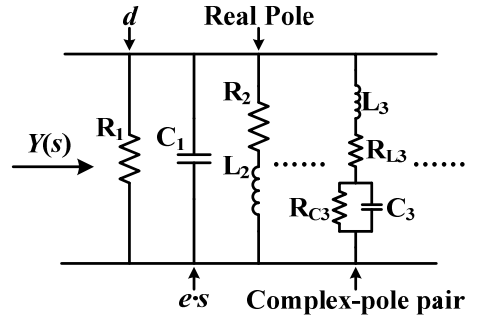

Fig. 6. Equivalent circuit synthesis for the Foster expansion of $Y(s)$.

equivalent circuit corresponding to the admittance $Y(s)$ in Foster expansion is illustrated.

As seen, the proposed IRFA together with the equivalent circuit synthesis method enable generating a highly accurate HF model from a measured impedance (or admittance) with flexibility. Based the IRFA and the circuit synthesis method, the HF model for $\mathrm{CM}$ chokes will be introduced in the following section.

\section{IRFA ADAPTED HF MODEL OF CM CHOKES AND EXTRACTION PROCEDURE}

\section{A. Proposed HF Model of CM chokes}

The proposed HF model of CM chokes is shown in Fig. 7. It should be noted that the parasitic capacitances are assumed to be lossless. Besides, the capacitances $C_{\mathrm{s}}$ in Fig. 2(b) are discarded in our model since $C_{\mathrm{e}}, C_{\mathrm{p}}$ and $C_{\mathrm{c}}$ are sufficient for describing the electrostatic behavior of a strongly coupled two-winding transformer [13]. Instead of using a heuristic method to identify the leakage impedance $Z_{1}$ and the magnetizing impedance $Z_{2}$, the topologies of $Z_{1}$ and $Z_{2}$ of the proposed model is represented by the equivalent circuit in Fig. 6 , which are determined by the results of the IRFA method. It is to be noted that the choice of the topologies follows strict

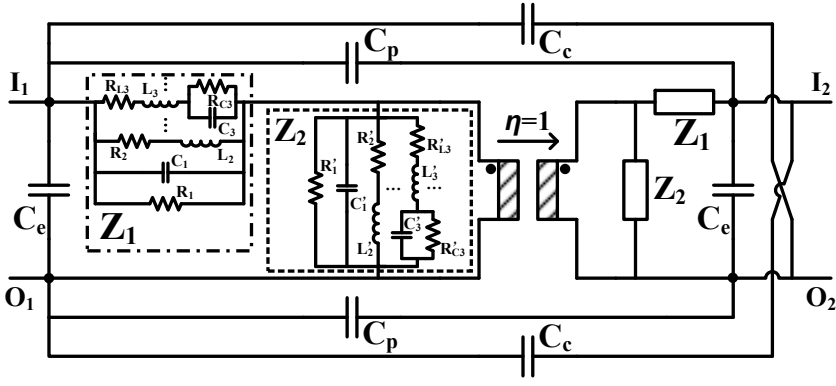

Fig. 7. Proposed HF model for CM chokes.

mathematical calculation so that the final equivalent circuit is guaranteed to be accurate over a wide frequency range.

\section{B. Extraction Procedure}

The extraction of the proposed HF model shown in Fig. 7 begins with three specific impedance measurements $T_{3}, T_{0}$ and $\mathrm{T}_{2}$, as shown in Table II. The IRFA method is then applied to extract the equivalent circuit for the impedances $Z_{\mathrm{T} 3}, Z_{\mathrm{T} 0}$ and $Z_{\mathrm{T} 2}$. Note that the measurement $\mathrm{T}_{1}$ in Fig. 3 is discarded since $C_{\mathrm{s}}$ is removed from the model. Moreover, the measurement $\mathrm{T}_{4}$ used in [15] is not necessary in the proposed procedure because $\mathrm{T}_{2}$ can provide enough information to extract the magnetizing impedance $Z_{2}$. In order to validate the proposed method and the extraction procedure, it is applied to model a planar CM choke with 8 turns on each winding. First, impedance measurements are carried out with an HP4294A precision impedance analyzer. The configurations $\mathrm{T}_{3}, \mathrm{~T}_{0}$ and $\mathrm{T}_{2}$ are treated successively, as described in the following parts. Configuration $\mathrm{T}_{3}$ represents a capacitive configuration which gives the value of $C_{\mathrm{c}}+C_{\mathrm{p}}$. The admittance $Y_{\mathrm{T} 3}=1 / Z_{\mathrm{T} 3}$ is fitted with the expression

$$
Y_{T 3}=1 / Z_{T 3}=d+e \cdot s
$$




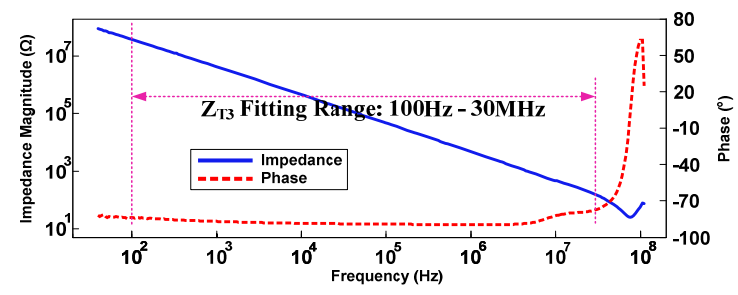

(a)

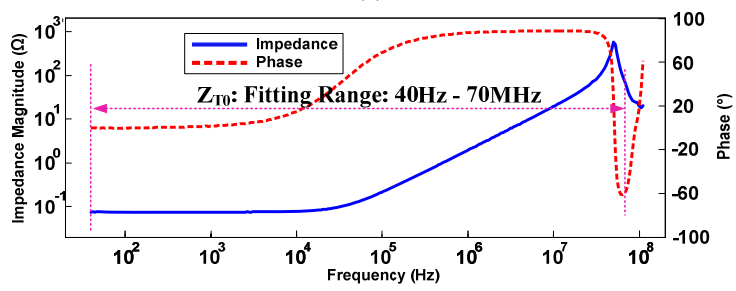

(b)

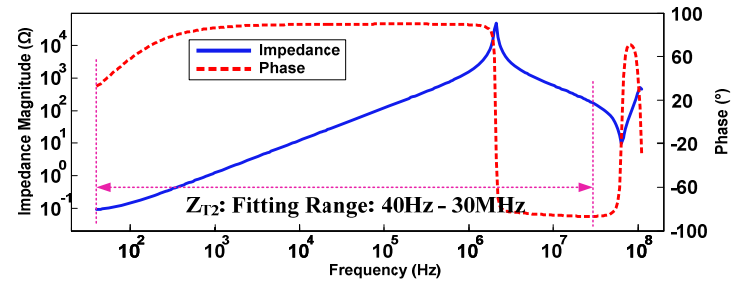

(c)

Fig. 8. Measured impedances for the extraction. (a) $Z_{\mathrm{T} 3}$. (b) $Z_{\mathrm{T} 0}$. (c) $Z_{\mathrm{T} 2}$.

where $d$ denotes the dielectric losses and $e$ denotes the capacitance $2\left(C_{\mathrm{c}}+C_{\mathrm{p}}\right)$. The fitting starts from $100 \mathrm{~Hz}$ to guarantee a good measurement precision of capacitances. As the capacitance model is an $R$ - $C$ parallel cell, the fitting stops at $30 \mathrm{MHz}$ to exclude the resonance, as shown in Fig. 8(a). The loss term $d$ is neglected according to the lossless capacitance assumption. The extracted result for $2\left(C_{\mathrm{c}}+C_{\mathrm{p}}\right)$ is $29.98 \mathrm{pF}$.

Configuration $\mathrm{T}_{0}$ is a flux-subtracting configuration so only the leakage impedance $Z_{\mathrm{l}} / 2$ and the capacitance $2\left(C_{\mathrm{e}}+C_{\mathrm{p}}\right)$ are concerned. Again, to facilitate the circuit synthesis, the admittance $Y_{\mathrm{T} 0}=1 / Z_{\mathrm{T} 0}$ is treated in the IRFA. In fact, the capacitance $2\left(C_{\mathrm{e}}+C_{\mathrm{p}}\right)$ corresponds the $e \cdot s$ term in Fig. 6 , so the rational function $Y_{\mathrm{T} 0}(s)$ represented in form of (1) must fulfill: $m-n=1$. In order to obtain a simple and accurate equivalent circuit, the values of $m$ and $n$ should be lowest possible but still guarantee an acceptable accuracy. In this case, we choose $m=6$ and $n=5$ after a few trials. In order to extract the DC resistance as well as the parasitic capacitance $2\left(C_{\mathrm{e}}+C_{\mathrm{p}}\right)$, the fitting frequency range covers from $40 \mathrm{~Hz}$ to $70 \mathrm{MHz}$, as illustrated in Fig. 8(b). The fitting results for $Y_{\mathrm{T} 0}(s)$ are listed in Table III.

It can be seen from Table III that the extracted $e_{\mathrm{T} 0}$ is negative, resulting in a negative capacitance. The latter is not allowed because it will cause simulation instabilities [23]. In fact, the phase plot of $Z_{\mathrm{T} 0}$ (see Fig. 8(b)) shows that this impedance is not capacitive beyond the resonance frequency $f_{r}$ $\approx 51 \mathrm{MHz}$. Around this frequency, the measured impedance $Z_{\mathrm{T} 0}$ is affected by distributed effect because the winding length of the studied CM choke is about $1 \mathrm{~m}$, which is
TABLE II

MEASUREMENT CONFIGURATIONS FOR THE PROPOSED EXTRACTION PROCEDURE

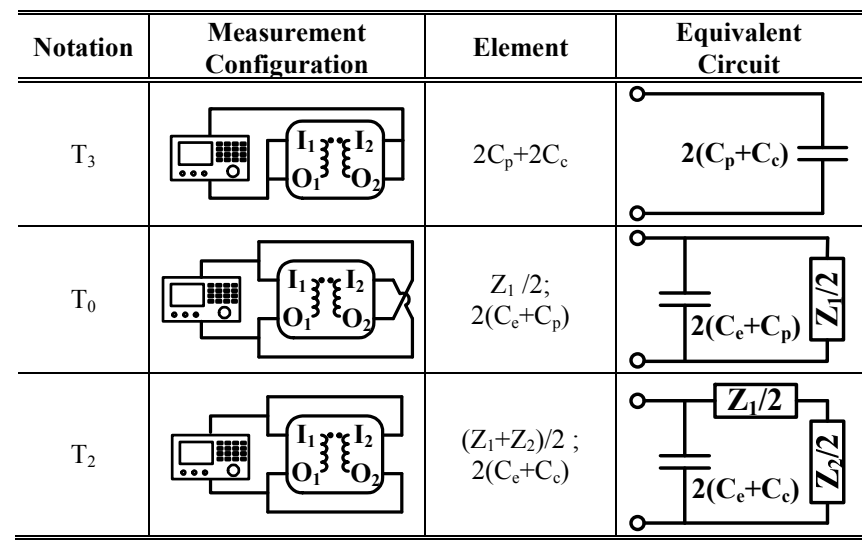

comparable to the wave length of the FR-4 based PCB structure at $f_{r}$. Therefore, using the simple parallel circuit shown in Table II to describe $Z_{\mathrm{T} 0}$ or $Y_{\mathrm{T} 0}$ gives rise to the negative capacitance. To obtain a positive capacitance, an approximation is performed. First, the complex pole-residue pair $\left(p_{\mathrm{T} 0}, r_{\mathrm{T} 0}\right)-\left(p_{\mathrm{T} 0}^{*}, r_{\mathrm{T} 0}^{*}\right), d_{\mathrm{T} 0}=3.0893 \mathrm{E}-3$ and $e_{\mathrm{T} 0}=-3.2803 \mathrm{E}-$ 12 terms of $Y_{\mathrm{T} 0}(s)$ in Table III are recombined together to form a new admittance called $Y_{\mathrm{CT} 0}(s)$

$$
Y_{\mathrm{CT} 0}(s)=d_{\mathrm{T} 0}+e_{\mathrm{T} 0} \cdot s+\frac{r_{\mathrm{T} 0}}{s-p_{\mathrm{T} 0}}+\frac{r_{\mathrm{T} 0}^{*}}{s-p_{\mathrm{T} 0}^{*}} .
$$

Indeed, the "true" parasitic capacitance is merged in $Y_{\text {Ст0 }}(s)$. The expression (13) is then simplified using $Y_{\mathrm{CT} 0}^{\prime}(s)=d_{\mathrm{T} 0}^{\prime}$ $+e_{\mathrm{T} 0}^{\prime} s$ by equating the values of $Y_{\mathrm{CT} 0}(s)$ and $Y_{\mathrm{CT} 0}^{\prime}(s)$ at the resonance frequency $s_{\mathrm{r}}=j 2 \pi f_{r}$, as given by

$$
d_{\mathrm{T} 0}+e_{\mathrm{T} 0} \cdot s_{r}+\frac{r_{\mathrm{T} 0}}{s_{r}-p_{\mathrm{T} 0}}+\frac{r_{\mathrm{T} 0}^{*}}{s_{r}-p_{\mathrm{T} 0}^{*}}=d_{\mathrm{T} 0}^{\prime}+e_{\mathrm{T} 0}^{\prime} \cdot s_{r} .
$$

The obtained results are: $e_{\mathrm{T} 0}^{\prime}=3.22 \mathrm{E}-11$ and $d_{\mathrm{T} 0}^{\prime}=1.48 \mathrm{E}-03$, which can be further represented by an $R-C$ parallel cell. With this simplification, the positive parasitic capacitance $2\left(C_{\mathrm{e}}+C_{\mathrm{p}}\right)$ and its parallel resistance is obtained. As the complex pole pair is thrown away during the approximation, the fitting precision of the real part of $Z_{\mathrm{T} 0}$ is impaired at high frequencies, since the complex poles are indispensable for obtaining high fitting precision around the resonance frequency, as shown in Fig. 9(a). However, trade-offs have to be accepted with this lumped-element equivalent circuit.

The final circuit representation for impedance $Z_{\mathrm{T} 0}$ is shown in Fig. 10(b). The impedance $Z_{1} / 2$ is contained in the dashed box. The elements of the $R-L$ cells are calculated with the real pole-residue pairs listed in Table III. These components can be interpreted as the Partial Element Equivalent Circuits (PEEC) for modeling the eddy current effect.

Configuration $\mathrm{T}_{2}$ is used as the last step of the procedure to identify the equivalent circuit of $Z_{\mathrm{T} 2}$. A rough calculation is first performed at $100 \mathrm{kHz}$ to estimate the $\mathrm{CM}$ inductance with $\operatorname{Im}\left(Z_{\mathrm{T} 2}\right)=\omega L_{\mathrm{CM}} / 2$, giving $L_{\mathrm{CM}}=382.4 \mu \mathrm{H}$. Similar to 
TABLE III

FITTING RESUlts FOR THE PLANAR CM CHOKE

\begin{tabular}{|c|c|c|c|c|c|c|c|}
\hline \multicolumn{3}{|c|}{$Y_{\mathrm{T} 0}(s)=1 / Z_{\mathrm{T} 0}(s)$} & \multicolumn{2}{|c|}{$Y_{\mathrm{T} 2}(s)=1 / Z_{\mathrm{T} 2}(s)$} & \multicolumn{3}{|c|}{$2 Y_{2}(s)=2 / Z_{2}(s)$} \\
\hline \multicolumn{3}{|c|}{$\bar{d}_{\mathrm{T} 0}=3.0893 \mathrm{E}-3 ; e_{\mathrm{T} 0}=-3.2803 \mathrm{E}-12$} & \multicolumn{2}{|c|}{ 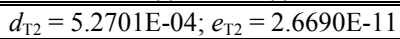 } & \multicolumn{3}{|c|}{$\bar{c}_{2}=4.9593 \mathrm{E}-04 ; e_{2}=8.2615 \mathrm{E}-14$} \\
\hline Poles & Residues & $F_{i}$ & Poles & Residues & Poles & Residues & $F_{i}$ \\
\hline$-7.3080 \mathrm{E}+07$ & $3.6178 \mathrm{E}+06$ & \multirow{4}{*}{0.05} & $-1.6659 \mathrm{E}+07$ & $-2.1578 \mathrm{E}+02$ & $-1.6679 \mathrm{E}+07$ & $-2.1486 \mathrm{E}+02$ & \multirow{4}{*}{$4.0 \mathrm{E}-5$} \\
\hline$+i 5.5497 \mathrm{E}+08$ & $+i 1.2356 \mathrm{E}+06$ & & $+i 1.8259 \mathrm{E}+07$ & $+i 6.3580 \mathrm{E}+02$ & $+i 1.8265 \mathrm{E}+07$ & $+i 6.3909 \mathrm{E}+02$ & \\
\hline$-7.3080 \mathrm{E}+07$ & $3.6178 \mathrm{E}+06$ & & $-1.6659 \mathrm{E}+07$ & $-2.1578 \mathrm{E}+02$ & $-1.6679 \mathrm{E}+07$ & $-2.1486 \mathrm{E}+02$ & \\
\hline$-i 5.5497 \mathrm{E}+08$ & $-i 1.2356 \mathrm{E}+06$ & & $-i 1.8259 \mathrm{E}+07$ & $-i 6.3580 \mathrm{E}+02$ & $-i 1.8265 \mathrm{E}+07$ & $-i 6.3909 \mathrm{E}+02$ & \\
\hline$-2.8458 \mathrm{E}+07$ & $1.0814 \mathrm{E}+05$ & $3.8 \mathrm{E}-3$ & $-2.2332 \mathrm{E}+08$ & $-1.0501 \mathrm{E}+05$ & $-2.1616 \mathrm{E}+08$ & $-9.4903 \mathrm{E}+04$ & $4.4 \mathrm{E}-4$ \\
\hline$-1.8105 \mathrm{E}+06$ & $1.6114 \mathrm{E}+05$ & 0.09 & $-2.0256 \mathrm{E}+05$ & $4.2972 \mathrm{E}+01$ & $-2.0165 \mathrm{E}+05$ & $4.2780 \mathrm{E}+01$ & $2.1 \mathrm{E}-5$ \\
\hline$-2.3224 \mathrm{E}+05$ & $3.0680 \mathrm{E}+06$ & 13.2 & $-4.1835 \mathrm{E}+02$ & $5.2350 \mathrm{E}+03$ & $-2.4875 \mathrm{E}+01$ & $5.2440 \mathrm{E}+03$ & 211 \\
\hline
\end{tabular}

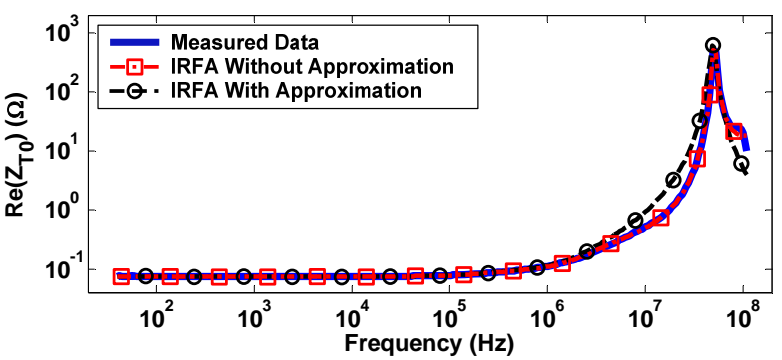

(a)

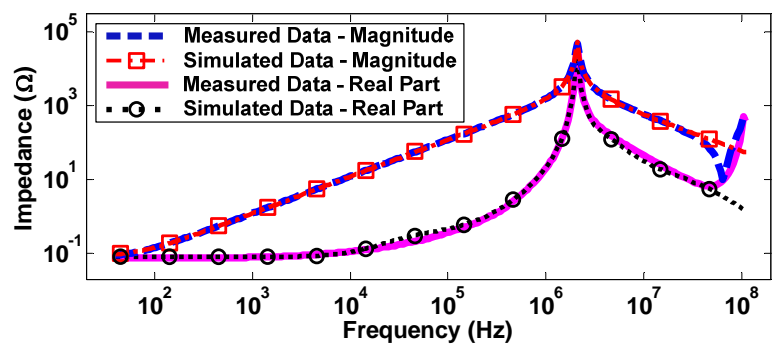

(b)

Fig. 9. Equivalent circuit extraction using IRFA. (a) $Z_{\mathrm{T} 0}$. (b) $Z_{\mathrm{T} 2}$.

$Z_{\mathrm{T} 0}$, the admittance form $Y_{\mathrm{T} 2}$ is processed for the fitting using a rational function with $\mathrm{m}=6$ and $\mathrm{n}=5$. As shown in Fig. 8 (c), the fitting stops at $30 \mathrm{MHz}$, just before the second resonance that cannot be modeled by the lumped-element equivalent circuit in Fig. 7. The fitting results are shown in Table III. According to the equivalent circuit $\mathrm{T}_{2}$ in Table II, $e_{\mathrm{T} 2}$ corresponds to the value of the parasitic capacitance $2\left(C_{\mathrm{e}}+C_{\mathrm{c}}\right)$ whereas the remaining parts (i.e. $d_{\mathrm{T} 2}$, real poles and complex poles) represent the admittance $2\left(Z_{1}+Z_{2}\right)^{-1}$. With $Z_{1}$ being identified [see Fig. 10(b)], the magnetizing impedance $Z_{2}$ is calculated by subtracting the contribution of $Z_{1}$. The IRFA is applied again to get the pole-residue information of the admittance $Y_{2}(s)=1 / Z_{2}(s)$, as listed in Table III. Note that a real pole $p=-2.1616 \mathrm{E}+08$ with negative residue $r=$ $9.4903 \mathrm{E}+04$ is extracted. This negative pole-residue is treated with the method presented in Table I, resulting in a positive $R(2.28 \mathrm{k} \Omega)-C(2.03 \mathrm{pF})$ series branch, which is interpreted as the nonmagnetic HF losses in the ferrite material due to the capacitive isolation of the grains [23]. The final equivalent circuit of $Z_{2} / 2$ and $Z_{\mathrm{T} 2}$ is shown in Fig. $10(\mathrm{c})$. In $Z_{2}$, the $L(190$ $\mu \mathrm{H})-R(4 \mathrm{~m} \Omega)$ branch presents the dominant pole according the definition: a pole $p_{i}$ with its residue $r_{i}$ is called dominant

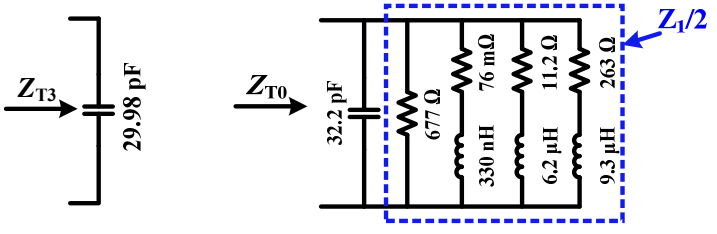

(a)

(b)

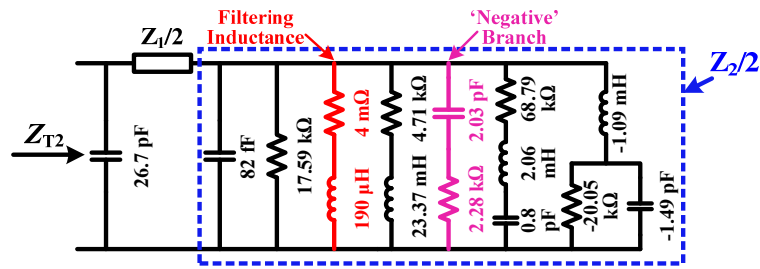

(c)

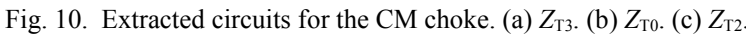

pole if its $F_{i}=\left|r_{i} / \operatorname{Re}\left(p_{i}\right)\right|$ is much larger than the other poles. The values $F_{i}$ for the poles of $Z_{\mathrm{T} 0}$ and $Z_{2}$ are listed in Table III and the $F_{i}$ of the $L(190 \mu \mathrm{H})-R(4 \mathrm{~m} \Omega)$ branch is much larger than the others. Therefore, it has the most important influence in $Z_{2}$. The $190 \mu \mathrm{H}$ inductance is in fact the $\mathrm{CM}$ filtering inductance because it is half the $\mathrm{CM}$ inductance $\mathrm{L}_{\mathrm{CM}}$ estimated from $Z_{\mathrm{T} 2}$. The simulated impedance of $Z_{\mathrm{T} 2}$ is compared with measured data in Fig. 9(b) in terms of real part and magnitude. As seen, the simulated results agree well with the measurements up to $30 \mathrm{MHz}$.

With the parasitic capacitances obtained from the three configurations, three linear equations expressed in (15) can be established, giving the values of the stray capacitances: $C_{\mathrm{c}}=$ $6.1 \mathrm{pF}, C_{\mathrm{e}}=7.2 \mathrm{pF}$ and $C_{\mathrm{p}}=8.9 \mathrm{pF}$.

$$
\left\{\begin{array}{l}
2 C_{\mathrm{c}}+2 C_{\mathrm{p}}=30 \mathrm{pF} \\
2 C_{\mathrm{e}}+2 C_{\mathrm{p}}=32.2 \mathrm{pF} \\
2 C_{\mathrm{e}}+2 C_{\mathrm{c}}=26.7 \mathrm{pF}
\end{array} .\right.
$$

Till now the HF model for the studied planar CM choke is established. The obtained results will be validated by experimental tests in next section.

\section{EXPERIMENTAL VERIFICATION AND DISCUSSION}

\section{A. Experimental Verification}

To validate the HF model and the procedure, the planar CM choke is first examined by impedance measurements. Two 


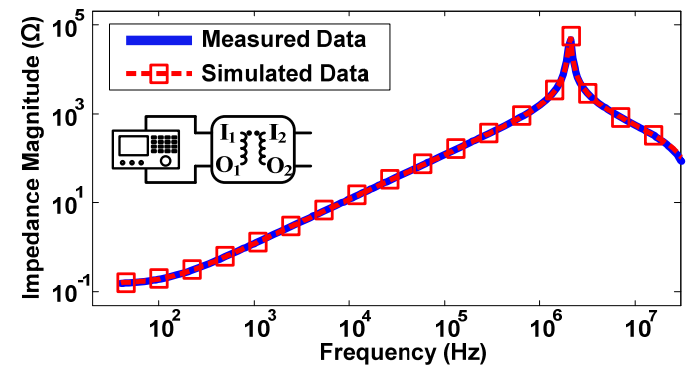

(a)

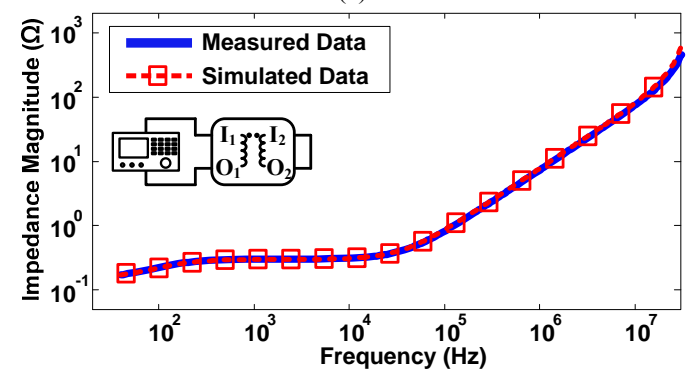

(b)

Fig. 11. Comparison between the measured and simulated impedances of the HF model. (a) Open circuit test- $Z_{\mathrm{O}}$. (b) Short circuit test- $Z_{\mathrm{SC}}$.

measurement configurations are chosen: open circuit $Z_{\mathrm{O}}$ and short circuit $Z_{\mathrm{SC}}$. These two configurations correspond to extreme loaded impedances for the component. Using SPICE, an $\mathrm{AC}$ analysis from $40 \mathrm{~Hz}$ to $30 \mathrm{MHz}$ (Log Sweep) is performed on the identified HF model and the simulation results are compared with measurements data, as shown in Fig. 11. It can be seen that the simulated curves match closely with the measured ones from $40 \mathrm{~Hz}$ to $30 \mathrm{MHz}$.

As there are many small-valued elements in the model, the sensitivity of the model should be examined. Some qualitative results are given for this analysis.

1) The three parasitic capacitances $C_{\mathrm{e}}, C_{\mathrm{p}}$ and $C_{\mathrm{c}}$ affect the resonance frequency and the response beyond the resonance.

2) In $Z_{1}$ and $Z_{2}$, the branches of dominant poles determine the behavior of the model on the inductive region. The model is less sensitive to the parameters in the braches of non-dominant poles.

3) The impedance around the resonance varies rapidly so they are sensitive to the dominant poles branches, the complex pole-pairs branches and parasitic capacitances.

In a word, the model is quite robust because the behavior of the model does not change abruptly due to small variations of some parameters. However, cares should always be taken during the measurements and the fitting process to guarantee a good accuracy of the extracted model.

Next, an EMI filter using the topology shown in Fig. 1 is realized with the planar CM choke, as shown in Fig. 12(a). The leakage of the CM choke acts as differential mode (DM) inductors. The impedance of the CM capacitors $(4.7 \mathrm{pF})$ and the $\mathrm{DM}$ capacitor $(68 \mathrm{nF})$ are measured by impedance analyzer. The equivalent circuit for the capacitors is given in Fig. 12(b) and their impedance $Z_{C}(s)$ can be expressed by:

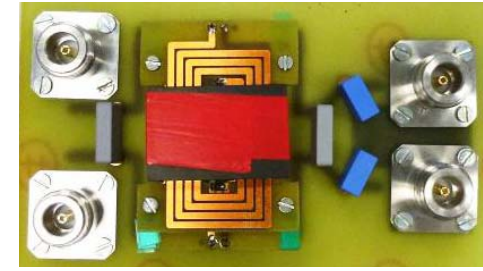

(a)

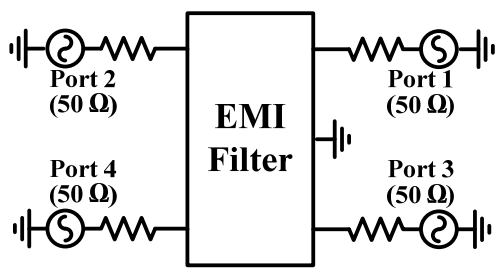

(c)

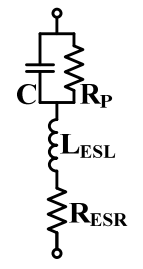

(b)

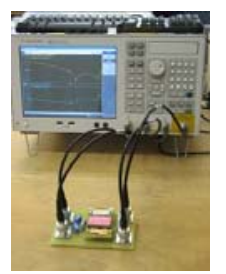

(d)
Fig. 12. IL measurements of the EMI filter. (a) EMI filter for test. (b) Equivalent circuit of capacitors. (c) Circuit of the measurement. (d) Measurement setup.

$$
Z_{C}(s)=R_{E S R}+s L_{E S L}+\frac{R_{P}}{s C R_{P}+1}
$$

where $R_{E S R}$ stands for equivalent series resistance and $L_{E S L}$ for equivalent series resistance. To get the values of the parameters, (16) can be fitted by the IRFA method using a rational function (1) with $m=2$ and $n=1$. The IL of the filter is measured on an Agilent 5071C network analyzer using a four-port S parameter measurement method [9] [see Fig. 12(c) and (d)]. The $\mathrm{S}$ parameter matrix $\left[S_{i j}, i, j=1,2,3,4\right]$ is measured from $100 \mathrm{kHz}$ to $30 \mathrm{MHz}$ with logarithmic sweep. Postprocessing of this matrix is performed to obtain the mixedmode $\mathrm{S}$ parameter $S_{\mathrm{cc} 21}$ and $S_{\mathrm{dd} 21}$ through the following equations:

$$
\left\{\begin{array}{l}
S_{\mathrm{cc} 21}=\frac{1}{2}\left(S_{21}+S_{23}+S_{41}+S_{43}\right) \\
S_{\mathrm{dd} 21}=\frac{1}{2}\left(S_{21}-S_{23}-S_{41}+S_{43}\right)
\end{array}\right.
$$

where $S_{\mathrm{cc} 21}$ and $S_{\mathrm{dd} 21}$ give the CM and DM insertion loss for the EMI filter, respectively [9]. Moreover, a four-port $S$ parameter simulation is performed over the same frequency range with the same configuration as Fig. 12(c). The simulated CM IL $S_{\mathrm{cc} 21 \_ \text {sim }}$ and DM IL $S_{\mathrm{dd} 21 \_ \text {sim }}$ are deduced by (17) as well. The comparison between the measured ILs and the simulated ones are presented in Fig. 13. It can be seen that the simulated result closely matches the measurement for common mode. However, large difference at HF is observed for the differential mode. This is due to the parasitic coupling effects among the choke, capacitors and trace loop [4], [11], which are not considered in this model. However, this is out of the scope of this paper.

\section{B. Discussion}

Through the IRFA method, accurate models for CM chokes can be built from impedance measurements. However, several important aspects of the model and the procedure should be addressed.

1) Passivity: As the whole extraction procedure treats passive magnetic components, the passivity issues have to be 


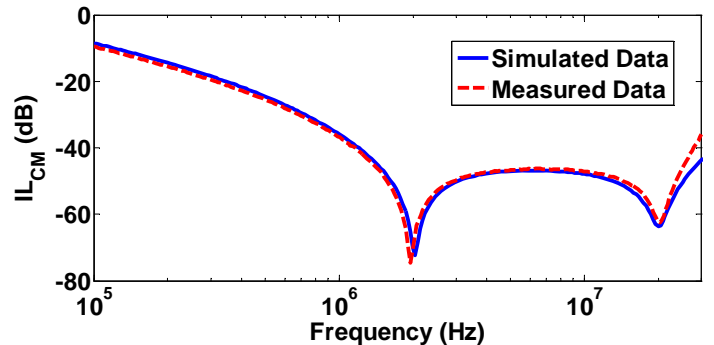

(a)

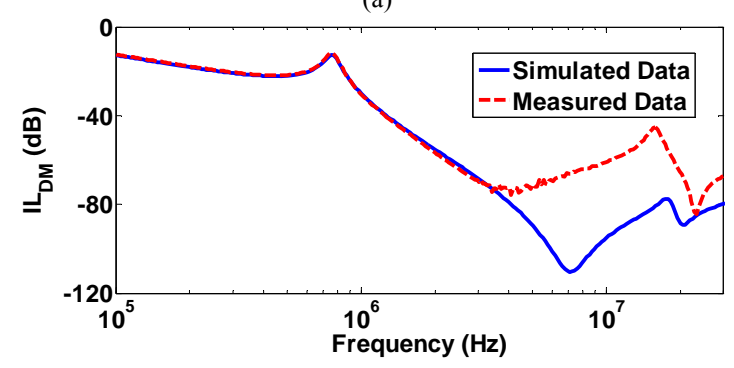

(b)

Fig. 13. Comparison of simulated and measured IL. (a) CM. (b) DM.

considered. The passivity of a component requires that the component be dissipative in energy. However, the IRFA method cannot guarantee the passivity of the calculated rational function. During the past ten years, many works have been reported on the passivity verification and enforcement techniques [24], [25]. In this paper, all the extracted results are verified to be passive using the method of [24]. In practice, as $\mathrm{CM}$ chokes are naturally dissipative in HF due to core losses and copper losses, the passivity is usually respected if fitting precision is good enough.

2) Distributed effect: Due to the distributed effect, manual adjustments are needed to avoid negative elements, which require some experience about the equivalent circuit synthesis. This is the limit of using a lumped-element equivalent circuit model. The model presented in this paper is effective up to 30 $\mathrm{MHz}$, which corresponds to the frequency range of interest for the conducted emissions. Nonetheless it is necessary to consider the distributed effect to cover frequencies beyond 30 $\mathrm{MHz}$, which requires implementing a distributed circuit model.

3) Saturation: CM chokes always work under flux bias which will cause saturation effect [26]. The proposed model is established on the basis of small signal measurements. Therefore, the error of the model will increase as the current increases. However, the primary focus of the paper is to propose a small signal HF model of CM chokes that can be systematically synthesized. As the first step, the small signal model is now available and it can then be coupled with nonlinear cell that accounts for the saturation of the magnetic material, which is the objective of a future study.

\section{CONCLUSION}

In this paper, a HF equivalent circuit model of CM chokes used in EMI filter is proposed incorporating a systematic extraction procedure. The procedure uses an iterative rational function approximation method to extract pole-residue form expressions from impedance measurements. Based on the pole-residue information, a systematic synthesis method is applied to generate the equivalent circuit. The proposed procedure gives accurate models in a short time. This procedure is applied on a planar CM choke and is validated by simulations and measurements.

\section{APPENDIX}

According to the SK iteration, the denominator of the iteration $t-1 D^{[t-1]}\left(s_{k}\right)$ is divided at both sides of the equation (3) of the iteration $t$, resulting in (6) and

$$
\frac{N^{[t]}\left(s_{k}\right)\left(D^{[t-1]}\left(s_{k}\right)\right)^{*}}{\left|D^{[t-1]}\left(s_{k}\right)\right|^{2}}=\frac{Z_{\text {meas }}\left(s_{k}\right) D^{[t]}\left(s_{k}\right)\left(D^{[t-1]}\left(s_{k}\right)\right)^{*}}{\left|D^{[t-1]}\left(s_{k}\right)\right|^{2}}
$$

where the superscript ${ }^{*}$ denotes the complex conjugate. For magnetic components, the imaginary part of the impedance $(Z \approx \omega L)$ is much larger than its real part (losses) in the inductive region. This unbalance will lead to a low fitting quality for the real part during the least square process. In order to achieve a balanced fitting precision between the real part and the imaginary part, the real and imaginary parts of (18) are normalized respectively by $1 / \operatorname{Re}\left[Z^{[t-1]}\left(s_{k}\right)\right]$ and $1 / \operatorname{Im}\left[Z^{[t-1]}\left(s_{k}\right)\right]$ as weighting factors:

$$
\left\{\begin{array}{l}
\frac{1}{\operatorname{Re}\left[Z^{[t-1]}\left(s_{k}\right)\right]}=\frac{\left|D^{[t-1]}\left(s_{k}\right)\right|^{2}}{\operatorname{Re}\left[N^{[t-1]}\left(s_{k}\right)\left(D^{[t-1]}\left(s_{k}\right)\right)^{*}\right]} \\
\frac{1}{\operatorname{Im}\left[Z^{[t-1]}\left(s_{k}\right)\right]}=\frac{\left|D^{[t-1]}\left(s_{k}\right)\right|^{2}}{\operatorname{Im}\left[N^{[t-1]}\left(s_{k}\right)\left(D^{[t-1]}\left(s_{k}\right)\right)^{*}\right]}
\end{array}\right.
$$

Combining the SK iteration (18) with the weighting factors (19), the final formulations (7) and (8) are derived.

\section{REFERENCES}

[1] K. Mainali and R. Oruganti, "Conducted EMI Mitigation Techniques for Switch-Mode Power Converters: A Survey," IEEE Trans. Power Electron., vol. 25, pp. 2344-2356, Sep. 2010.

[2] Y. Maillet, R. Lai, S. Wang, F. Wang, R. Burgos, and D. Boroyevich, "High-Density EMI Filter Design for DC-Fed Motor Drives," IEEE Trans. Power Electron., vol. 25, pp. 1163-1172, May 2010.

[3] M. Hartmann, H. Ertl, and J. W. Kolar, "EMI Filter Design for a $1 \mathrm{MHz}$, $10 \mathrm{~kW}$ Three-Phase/Level PWM Rectifier, "IEEE Trans. Power Electron., vol.26, pp.1192-1204, Apr. 2011.

[4] S. Wang, F. C. Lee, D. Y. Chen, and W. G. Odendaal, "Effects of Parasitic Parameters on EMI Filter Performance," IEEE Trans. Power Electron., vol. 19, pp. 869-877, May 2004.

[5] M. Kovacic, Z. Hanic, S. Stipetic, S. Krishnamurthy and D. Zarko, "Analytical Wideband Model of a Common-Mode Choke," IEEE Trans. Power Electron., vol.27, pp.3173-3185, Jul. 2012.

[6] X. Wu, D. Xu, Z. Wen, Y. Okuma and K. Mino, "Design, Modeling, and Improvement of Integrated EMI Filter With Flexible Multilayer Foils," IEEE Trans. Power Electron., vol.26, pp.1344-1354, May 2011.

[7] V. Tarateeraseth, B. Hu, K. Y. See, and F. G. Canavero, "Accurate Extraction of Noise Source Impedance of an SMPS Under Operating Conditions," IEEE Trans. Power Electron., vol. 25, pp. 111-117, Jan. 2010 . 
[8] P. Kong, Y. Jiang, and F. C. Lee, "Common Mode EMI Noise Characteristics of Low-Power AC-DC Converters," IEEE Trans. Power Electron., vol.27, pp.731-738, Feb. 2012.

[9] K. S. Kostov and J. J. Kyyrä, "Insertion loss in terms of four-port network parameters," IET Science, Measurement \& Technology, vol. 3, pp. 208-216, May 2009.

[10] A.-M. Sánchez, A. Pérez, J. R. Regué, M. Ribó, P. Rodríguez-Cepeda, and F. J. Pajares, "A Modal Model of Common-Mode Chokes for Conducted Interference Prediction," IEEE Trans. Electromagn. Compat., vol. 52, pp. 749-752, Aug. 2010.

[11] S. Wang, F. C. Lee, and W. G. Odendaal, "Characterization and Parasitic Extraction of EMI Filters Using Scattering Parameters," IEEE Trans. Power Electron., vol. 20, pp. 502-510, Mar. 2005.

[12] X. Margueron and J.-P. Keradec, "Identifying the Magnetic Part of the Equivalent Circuit of n-Winding Transformers," IEEE Trans. Instrum. and Meas., vol.56, pp.146-152, Feb. 2007.

[13] A. Schellmanns, K. Berrouche, and J. -P. Keradec, "Multiwinding transformers: a successive refinement method to characterize a general equivalent circuit," IEEE Trans. Instrum. Meas., vol.47, pp.1316-1321, Oct. 1998.

[14] J. -L. Kotny, X. Margueron and N. Idir, "High frequency modeling method of EMI filters," in 2009 Proc. IEEE ECCE Conf., pp.16711678.

[15] J. -L. Kotny, X. Margueron, and N. Idir, "High Frequency Model of the coupled inductors used in EMI Filters," IEEE Trans. Power Electron., vol. 27, pp. 2805-2812, Jun. 2012.

[16] S. Skibin and I. Stevanovic, "Behavioral circuit modeling of chokes with multi-resonances using genetic algorithm," in 2011 Proc. IEEE Symp. EMC, pp. 454-458.

[17] W. Tan, C. Cuellar, X. Margueron, and N. Idir, "Automatic Identification of Magnetic Component Equivalent Circuits Using Impedance Measurements," in 2011 Proc. IEEE Instrum. and Meas. Technol. Conf., pp.1317-1322, May 2011.

[18] M. Elzinga, K. Virga, L. Zhao, and J. L. Prince, "Pole-residue formulation for transient simulation of high-frequency interconnects using householder LS curve-fitting techniques," IEEE Trans. Adv. Packag., vol. 25, pp.142-147, May 2000.

[19] E.C. Levy, "Complex-curve fitting," IRE Trans. Automat. Contr., vol. AC-4, pp. 37-44, 1959.

[20] C. K. Sanathanan and J. Koerner, "Transfer function synthesis as a ratio of two complex polynomials," IEEE Trans. Autom. Control, vol. AC-8, pp.56, Jan. 1963.

[21] G. Antonini, "Spice equivalent circuits of frequency domain responses," IEEE Trans. Electromagn. Compat., vol. 45, pp.502-512, Aug. 2003.

[22] $\mathrm{Z}$. Ye and $\mathrm{Z}$. Yu, "Passive-assured rational function approach for compact modeling of on-chip passive components," in 2004 Proc. Int. Conf. Solid-State and Integrated Circuits Technol., pp. 217-220.

[23] A. Schellmanns, P. Fouassier, J.-P. Keradec, and J.-L. Schanen, "Equivalent circuits for transformers based on one-dimensional propagation: accounting for multilayer structure of windings and ferrite losses," IEEE Trans. Magn., vol.36, pp.3778-3784, Sep. 2000.

[24] B. Gustavsen and A. Semlyen, "Enforcing passivity for admittance matrices approximated by rational functions," IEEE Trans. Power Systems, vol. 16, pp.97-104, Jan. 2001.

[25] S. Grivet-Talocia and A. Ubolli, "A Comparative Study of Passivity Enforcement Schemes for Linear Lumped Macromodels," IEEE Trans. Adv. Packag., vol. 31, pp.673-683, Nov. 2008.

[26] N. Zhu, J. Kang, D. Xu, B. Wu, and Y. Xiao, “An Integrated AC Choke Design for Common-Mode Current Suppression in Neutral-Connected Power Converter Systems," IEEE Trans. Power Electron., vol.27, pp.1228-1236, Mar. 2012. 